\title{
Matching polytopes, toric geometry, and the totally non-negative Grassmannian
}

\author{
Alexander Postnikov • David Speyer • \\ Lauren Williams
}

Received: 25 April 2008 / Accepted: 17 October 2008 / Published online: 3 November 2008 (C) Springer Science+Business Media, LLC 2008

\begin{abstract}
In this paper we use toric geometry to investigate the topology of the totally non-negative part of the Grassmannian, denoted $\left(G r_{k, n}\right)_{\geq 0}$. This is a cell complex whose cells $\Delta_{G}$ can be parameterized in terms of the combinatorics of plane-bipartite graphs $G$. To each cell $\Delta_{G}$ we associate a certain polytope $P(G)$. The polytopes $P(G)$ are analogous to the well-known Birkhoff polytopes, and we describe their face lattices in terms of matchings and unions of matchings of $G$. We also demonstrate a close connection between the polytopes $P(G)$ and matroid polytopes. We use the data of $P(G)$ to define an associated toric variety $X_{G}$. We use our technology to prove that the cell decomposition of $\left(G r_{k, n}\right)_{\geq 0}$ is a CW complex, and furthermore, that the Euler characteristic of the closure of each cell of $\left(G r_{k, n}\right)_{\geq 0}$ is 1 .
\end{abstract}

Keywords Total positivity - Grassmannian - CW complexes · Birkhoff polytope Matching $\cdot$ Matroid polytope $\cdot$ Cluster algebra

Alexander Postnikov was supported in part by NSF CAREER Award DMS-0504629. David Speyer was supported by a research fellowship from the Clay Mathematics Institute. Lauren Williams was supported in part by the NSF.

A. Postnikov · D. Speyer

Department of Mathematics, Massachusetts Institute of Technology, 77 Massachusetts Avenue, Cambridge, MA 02139, USA
A. Postnikov
e-mail: apost@math.mit.edu
D. Speyer
e-mail: speyer@math.mit.edu

L. Williams $(\bowtie)$

Department of Mathematics, Harvard University, 1 Oxford Street, Cambridge, MA 02138, USA

e-mail: lauren@math.harvard.edu 


\section{Introduction}

The classical theory of total positivity concerns matrices in which all minors are nonnegative. While this theory was pioneered by Gantmacher, Krein, and Schoenberg in the 1930's, the past decade has seen a flurry of research in this area initiated by Lusztig [9-11]. Motivated by surprising connections he discovered between his theory of canonical bases for quantum groups and the theory of total positivity, Lusztig extended this subject by introducing the totally non-negative variety $\mathbb{G} \geq 0$ in an arbitrary reductive group $\mathbb{G}$ and the totally non-negative part $(\mathbb{G} / P)_{\geq 0}$ of a real flag variety $\mathbb{G} / P$.

Recently Postnikov [13] investigated the combinatorics of the totally non-negative part of a Grassmannian $\left(G r_{k, n}\right)_{\geq 0}$ : he established a relationship between $\left(G r_{k, n}\right)_{\geq 0}$ and certain planar bicolored graphs, producing a combinatorially explicit cell decomposition of $\left(G r_{k, n}\right)_{\geq 0}$. To each such graph $G$ he constructed a parameterization $\operatorname{Meas}_{G}$ of a corresponding cell of $\left(G r_{k, n}\right)_{\geq 0}$ by $\left(\mathbb{R}_{>0}\right)^{\# \text { Faces }(G)-1}$. In fact, this cell decomposition is a special case of a cell decomposition of $(\mathbb{G} / P)_{\geq 0}$ which was conjectured by Lusztig and proved by Rietsch [14], although that cell decomposition was described in quite different terms. Other combinatorial aspects of $\left(G r_{k, n}\right)_{\geq 0}$, and more generally of $(\mathbb{G} / P)_{\geq 0}$, were investigated by Marsh and Rietsch [12], Rietsch [15], and the third author [21, 22].

It is known that $(\mathbb{G} / P)_{\geq 0}$ is contractible [9] and it is conjectured that $(\mathbb{G} / P)_{\geq 0}$ with its cell decomposition is a regular CW complex homeomorphic to a ball. ${ }^{1}$ In [22], the third author proved the combinatorial analogue of this conjecture, proving that the partially ordered set (poset) of cells of $(\mathbb{G} / P)_{\geq 0}$ is in fact the poset of cells of a regular $\mathrm{CW}$ complex homeomorphic to a ball.

In this paper we give an approach to this conjecture which uses toric geometry to extend $\operatorname{Meas}_{G}$ to a map onto the closure of the corresponding cell of $\left(G r_{k, n}\right)_{\geq 0}$. Specifically, given a plane-bipartite graph $G$, we construct a toric variety $X_{G}$ and a rational map $m_{G}: X_{G} \rightarrow G r_{k, n}$. We show that $m_{G}$ is well-defined on the totally non-negative part of $X_{G}$ and that its image is the closure of the corresponding cell of $\left(G r_{k, n}\right)_{\geq 0}$. The totally non-negative part of $X_{G}$ is homeomorphic to a certain polytope (the moment polytope) which we denote $P(G)$, so we can equally well think of this result as a parameterization of our cell by $P(G)$. The restriction of $m_{G}$ to the toric interior of the non-negative part of $X_{G}$ (equivalently, to the interior of $P(G)$ ) is $\operatorname{Meas}_{G}$.

Our technology proves that the cell decomposition of the totally non-negative part of the Grassmannian is in fact a CW complex. While our map $m_{G}$ is well-defined on $\left(X_{G}\right)_{\geq 0}$ (which is a closed ball) and is a homeomorphism on the interior, in general $m_{G}$ is not a homeomorphism on the boundary of $\left(X_{G}\right)_{\geq 0}$; therefore this does not lead directly to a proof of the conjecture. However, we do obtain more evidence that the conjecture is true: using Williams' result [22] that the face poset of $(\mathbb{G} / P)_{\geq 0}$ is Eulerian, it follows that the Euler characteristic of the closure of each cell of $\left(G r_{k, n}\right)_{\geq 0}$ is 1 .

\footnotetext{
${ }^{1}$ This conjecture is parallel to a conjecture made by Fomin and Shapiro in [3].
} 
Table 1 How $G$ reflects $P(G)$

Plane-Bipartite graph $G$

Polytope $P(G)$

\#Faces $(G)-1$

Dimension of $P(G)$

Perfect orientations/almost perfect matchings

Vertices of $P(G)$

Equivalence classes of edges

Facets of $P(G)$

Lattice of elementary subgraphs

Lattice of faces of $P(G)$

The most elegant part of our story is how the combinatorics of the plane-bipartite graph $G$ reflects the structure of the polytope $P(G)$ and hence the structure of $X_{G}$. See Table 1 for some of these connections. The torus fixed points of $X_{G}$ correspond to perfect orientations of $G$, equivalently, to almost perfect matchings of $G$. The other faces of $X_{G}$ correspond to certain elementary subgraphs of $G$, that is, to unions of almost perfect matchings of $G$. Every face of $X_{G}$ is of the form $X_{G^{\prime}}$ for some plane-bipartite graph $G^{\prime}$ obtained by deleting some edges of $G$, and $m_{G}$ restricted to $X_{G^{\prime}}$ is $m_{G^{\prime}}$. It will follow from this that, for every face $Z$ of $X_{G}$, the interior of $Z$ is mapped to the interior of a cell of the totally non-negative Grassmannian with fibers that are simply affine spaces. We hope that this explicit description of the topology of the parameterization will be useful in studying the topology of $\left(G r_{k, n}\right)_{\geq 0}$.

The structure of this paper is as follows. In Section 2 we review the combinatorics of plane-bipartite graphs and perfect orientations. Next, in Section 3 we review toric varieties and their non-negative parts, and prove a lemma which is key to our CW complex result. We then, in Section 4, introduce the polytopes which will give rise to the toric varieties of interest to us. Using these polytopes, in Section 5 we make the connection between our polytopes $P(G)$ and matroid polytopes and explain the relation of our results to problems arising in cluster algebras and tropical geometry. In Section 6 we use these polytopes to prove that the cell decomposition of $\left(G r_{k, n}\right)_{\geq 0}$ is in fact a CW complex. In Section 7 we analyze the combinatorics of our polytopes in greater detail, giving a combinatorial description of the face lattice of $P(G)$ in terms of matchings and unions of matchings of $G$. Finally, in Appendix A, we calculate $f$ vectors, Ehrhart series, volumes, and the degrees of the corresponding toric varieties for a few small plane-bipartite graphs.

\section{The totally non-negative Grassmannian and plane-bipartite graphs}

In this section we review some material from [13]. We have slightly modified the notation from [13] to make it more convenient for the present paper.

Recall that the (real) Grassmannian $G r_{k, n}$ is the space of all $k$-dimensional subspaces of $\mathbb{R}^{n}$, for $0 \leq k \leq n$. An element of $G r_{k, n}$ can be viewed as a full-rank $k \times n$ matrix modulo left multiplications by nonsingular $k \times k$ matrices. In other words, two $k \times n$ matrices represent the same point in $G r_{k, n}$ if and only if they can be obtained from each other by row operations.

Let $\left(\begin{array}{c}{[n]} \\ k\end{array}\right)$ be the set of all $k$-element subsets of $[n]:=\{1, \ldots, n\}$. For $I \in\left(\begin{array}{c}{[n]} \\ k\end{array}\right)$, let $\Delta_{I}(A)$ denote the maximal minor of a $k \times n$ matrix $A$ located in the column set $I$. The map $A \mapsto\left(\Delta_{I}(A)\right)$, where $I$ ranges over $\left(\begin{array}{c}{[n]} \\ k\end{array}\right)$, induces the Plücker embedding $G r_{k, n} \hookrightarrow \mathbb{R P}\left(\begin{array}{l}n \\ k\end{array}\right)-1$. 
Definition 2.1 [13, Section 3] The totally non-negative Grassmannian $\left(G r_{k, n}\right)_{\geq 0}$ is the subset of the real Grassmannian $G r_{k, n}$ that can be represented by $k \times n$ matrices $A$ with all maximal minors $\Delta_{I}(A)$ non-negative.

For $\mathcal{M} \subseteq\left(\begin{array}{c}{[n]} \\ k\end{array}\right)$, the positive Grassmann cell $C_{\mathcal{M}}$ is the subset of the elements in $\left(G r_{k, n}\right)_{\geq 0}$ represented by all $k \times n$ matrices $A$ with the prescribed collection of maximal minors strictly positive $\Delta_{I}(A)>0$, for $I \in \mathcal{M}$, and the remaining maximal minors equal to zero $\Delta_{J}(A)=0$, for $J \notin \mathcal{M}$.

A subset $\mathcal{M} \subseteq\left(\begin{array}{c}{[n]} \\ k\end{array}\right)$ such that $C_{\mathcal{M}}$ is nonempty satisfies the base axioms of matroid. These special matroids are called positroids.

Clearly $\left(G r_{k, n}\right)_{\geq 0}$ is a disjoint union of the positive Grassmann cells $C_{\mathcal{M}}$. It was shown in [13] that each of these cells $C_{\mathcal{M}}$ is really a cell, that is, it is homeomorphic to an open ball of appropriate dimension $d$. Moreover, one can explicitly construct a parametrization $\mathbb{R}_{>0}^{d} \stackrel{\sim}{\rightarrow} C_{\mathcal{M}}$ using certain planar graphs, as follows.

Definition 2.2 A plane-bipartite graph is an undirected graph $G$ drawn inside a disk (considered modulo homotopy) with $n$ boundary vertices on the boundary of the disk, labeled $b_{1}, \ldots, b_{n}$ in clockwise order, as well as some colored internal vertices. These internal vertices are strictly inside the disk and are colored in black and white such that:

1. Each edge in $G$ joins two vertices of different colors.

2. Each boundary vertex $b_{i}$ in $G$ is incident to a single edge.

A perfect orientation $\mathcal{O}$ of a plane-bipartite graph $G$ is a choice of directions of its edges such that each black internal vertex $u$ is incident to exactly one edge directed away from $u$; and each white internal vertex $v$ is incident to exactly one edge directed towards $v$. A plane-bipartite graph is called perfectly orientable if it has a perfect orientation. Let $G_{\mathcal{O}}$ denote the directed graph associated with a perfect orientation $\mathcal{O}$ of $G$. The source set $I_{\mathcal{O}} \subset[n]$ of a perfect orientation $\mathcal{O}$ is the set of $i$ for which $b_{i}$ is a source of the directed graph $G_{\mathcal{O}}$. Similarly, if $j \in \bar{I}_{\mathcal{O}}:=[n] \backslash I_{\mathcal{O}}$, then $b_{j}$ is a sink of $\mathcal{O}$.

All perfect orientations of a fixed $G$ have source sets of the same size $k$ where $k-(n-k)=\sum \operatorname{color}(v)(\operatorname{deg}(v)-2)$. Here the sum is over all internal vertices $v$, $\operatorname{color}(v)=1$ for a black vertex $v$, and $\operatorname{color}(v)=-1$ for a white vertex; see [13]. In this case we say that $G$ is of type $(k, n)$.

Let us associate a variable $x_{e}$ with each edge of $G$. Pick a perfect orientation $\mathcal{O}$ of $G$. For $i \in I_{\mathcal{O}}$ and $j \in \bar{I}_{\mathcal{O}}$, define the boundary measurement $M_{i j}$ as the following power series in the $x_{e}^{ \pm 1}$ :

$$
M_{i j}:=\sum_{P}(-1)^{\operatorname{wind}(P)} x^{P}
$$

where the sum is over all directed paths in $G_{\mathcal{O}}$ that start at the boundary vertex $b_{i}$ and end at the boundary vertex $b_{j}$. The Laurent monomial $x^{P}$ is given by $x^{P}:=$ $\prod^{\prime} x_{e^{\prime}} / \prod^{\prime \prime} x_{e^{\prime \prime}}$, where the product $\prod^{\prime}$ is over all edges $e^{\prime}$ in $P$ directed from a white vertex to a black vertex, and the product $\prod^{\prime \prime}$ is over all edges $e^{\prime \prime}$ in $P$ directed from 
a black vertex to a white vertex. For any path $P$, let $\sigma_{1}, \sigma_{2}, \ldots, \sigma_{r} \in \mathbb{R} / 2 \pi \mathbb{Z}$ be the directions of the edges of $P$ (in order). Let $Q$ be the path through $\mathbb{R} / 2 \pi \mathbb{Z}$ which travels from $\sigma_{1}$ to $\sigma_{2}$ to $\sigma_{3}$ and so forth, traveling less then $\pi$ units of arc from each $\sigma_{i}$ to the next. The winding index wind $(P)$ is the number of times $Q$ winds around the circle $\mathbb{R} / 2 \pi \mathbb{Z}$, rounded to the nearest integer. The index wind $(P)$ is congruent to the number of self-intersections of the path $P$ modulo 2 .

Remark 2.3 Let us mention several differences in the notations given above and the ones from [13]. The construction in [13] was done for plabic graphs, which are slightly more general than the plane-bipartite graphs defined above. Edges in plabic graphs are allowed to join vertices of the same color. One can easily transform a plabic graph into a plane-biparte graph, without much change in the construction, by contracting edges which join vertices of the same color, or alternatively, by inserting vertices of different color in the middle of such edges.

Another difference is that we inverted the edge variables from [13] for all edges directed from a black vertex to a white vertex.

In [13] the boundary measurements $M_{i j}$ were defined for any planar directed graph drawn inside a disk. It was shown that one can easily transform any such graph into a plane-bipartite graph with a perfect orientation of edges that has the same boundary measurements.

Let $E(G)$ denote the edge set of a plane-bipartite graph $G$, and let $\mathbb{R}_{>0}^{E(G)}$ denote the set of vectors $\left(x_{e}\right)_{e \in E(G)}$ with strictly positive real coordinates $x_{e}$.

Lemma 2.4 [13, Lemma 4.3] The sum in each boundary measurement $M_{i j}$ evaluates to a subtraction-free rational expression in the $x_{e}$. Thus it gives a well-defined positive function on $\mathbb{R}_{>0}^{E(G)}$.

For example, suppose that $G$ has two boundary vertices, 1 and 2 and two internal vertices $u$ and $v$, with edges $a, b, c$ and $d$ running connecting $1 \rightarrow u, u \rightarrow v, v \rightarrow u$ and $v \rightarrow 2$. Then $M_{12}=a b d-a b c b d+a b c b c b d-\cdots=a b d /(1+b c)$. The sum only converges when $|b c|<1$ but, by interpreting it as a rational function, we can see that it gives a well defined value for any 4-tuple $(a, b, c, d)$ of positive reals.

If the graph $G_{\mathcal{O}}$ is acyclic then there are finitely many directed paths $P$, and wind $(P)=0$ for any $P$. In this case the $M_{i j}$ are clearly Laurent polynomials in the $x_{e}$ with positive integer coefficients, and the above lemma is trivial.

For a plane-biparte graph $G$ of type $(k, n)$ and a perfect orientation $\mathcal{O}$ with the source set $I_{\mathcal{O}}$, let us construct the $k \times n$ matrix $A=A(G, \mathcal{O})$ such that

1. The $k \times k$ submatrix of $A$ in the column set $I_{\mathcal{O}}$ is the identity matrix.

2. For any $i \in I_{\mathcal{O}}$ and $j \in \bar{I}_{\mathcal{O}}$, the minor $\Delta_{\left(I_{\mathcal{O}} \backslash\{i\}\right) \cup\{j\}}(A)$ equals $M_{i j}$.

These conditions uniquely define the matrix $A$. Its entries outside the column set $I_{\mathcal{O}}$ are $\pm M_{i j}$. The matrix $A$ represents an element of the Grassmannian $G r_{k, n}$. Thus, by Lemma 2.4, it gives the well-defined boundary measurement map

$$
\operatorname{Meas}_{G}: \mathbb{R}_{>0}^{E(G)} \rightarrow G r_{k, n}
$$


Clearly, the matrix $A(G, \mathcal{O})$ described above will be different for different perfect orientations $\mathcal{O}$ of $G$. However, all these different matrices $A(G, \mathcal{O})$ represent the same point in the Grassmannian $G r_{k, n}$.

Note that once we have constructed the matrix $A$, we can determine which cell of $\left(G r_{k, n}\right)_{\geq 0}$ we are in by simply noting which maximal minors are nonzero and which are zero.

Proposition 2.5 [13, Theorem 10.1] For a perfectly orientable plane-bipartite graph $G$, the boundary measurement map $\operatorname{Meas}_{G}$ does not depend on a choice of perfect orientation of $G$.

If we multiply the edge variables $x_{e}$ for all edges incident to an internal vertex $v$ by the same factor, then the boundary measurement $M_{i j}$ will not change. Let $V(G)$ denote the set of internal vertices of $G$. Let $\mathbb{R}_{>0}^{E(G) / V(G)}$ be the quotient of $\mathbb{R}_{>0}^{E(G)}$ modulo the action of $\mathbb{R}_{>0}^{V(G)}$ given by these rescalings of the $x_{e}$. If the graph $G$ does not have isolated connected components without boundary vertices ${ }^{2}$, then $\mathbb{R}_{>0}^{E(G) / V(G)} \simeq \mathbb{R}_{>0}^{|E(G)|-|V(G)|}$. The map Meas $\operatorname{Min}_{G}$ induces the map

$$
\tilde{M} \operatorname{eas}_{G}: \mathbb{R}_{>0}^{E(G) / V(G)} \rightarrow G r_{k, n},
$$

which (slightly abusing the notation) we also call the boundary measurement map.

Talaska [20] has given an explicit combinatorial formula for the maximal minors (also called Plücker coordinates) of such matrices $A=A(G, \mathcal{O})$. To state her result, we need a few definitions. A conservative flow in a perfect orientation $\mathcal{O}$ of $G$ is a (possibly empty) collection of pairwise vertex-disjoint oriented cycles. (Each cycle is self-avoiding, i.e. it is not allowed to pass through a vertex more than once.) For $|J|=\left|I_{\mathcal{O}}\right|$, a flow from $I_{\mathcal{O}}$ to $J$ is a collection of self-avoiding walks and cycles, all pairwise vertex-disjoint, such that the sources of these walks are $I_{\mathcal{O}} \backslash\left(I_{\mathcal{O}} \cap J\right)$ and the destinations are $J \backslash\left(I_{\mathcal{O}} \cap J\right)$. So a conservative flow can also be described as a flow from $I_{\mathcal{O}}$ to $I_{\mathcal{O}}$. The weight weight $(F)$ of a flow $F$ is the product of the weights of all its edges directed from the white to the black vertex, divided by the product of all its edges directed from the black to the white vertex. ${ }^{3}$ A flow with no edges has weight 1 .

Theorem 2.6 [20, Theorem 1.1] Fix a perfectly orientable $G$ and a perfect orientation $\mathcal{O}$. The minor $\Delta_{J}(A)$ of $A=A(G, \mathcal{O})$, with columns in position $J$, is given by

$$
\Delta_{J}=\left(\sum_{F} \operatorname{weight}(F)\right) /\left(\sum_{F^{\prime}} \operatorname{weight}\left(F^{\prime}\right)\right) .
$$

Here the sum in the numerator is over flows $F$ from $I_{\mathcal{O}}$ to $J$ and the sum in the denominator is over all conservative flows $F^{\prime}$.

\footnotetext{
${ }^{2}$ Clearly, we can remove all such isolated components without affecting the boundary measurements.

${ }^{3}$ Note that here we slightly differ from Talaska's convention in order to be consistent with our previous convention in defining $M_{i j}$.
} 
A point in the Grassmannian only depends on its Plücker coordinates up to multiplication by a common scalar. For our purposes, it is best to clear the denominators in Theorem 2.6, and give a purely (Laurent) polynomial formula:

Corollary 2.7 Using the notation of Theorem 2.6, the point of $G r_{k, n}$ corresponding to the row span of A has Plücker coordinates

$$
p_{J}:=\left(\sum_{F} \text { weight }(F)\right)
$$

where the sum is over flows $F$ from $I_{\mathcal{O}}$ to $J$.

Theorem 2.6 implies that the image of the boundary measurement map $\tilde{M} e a s_{G}$ lies in the totally non-negative Grassmannian $\left(G r_{k, n}\right)_{\geq 0}$. Moreover, the image is equal to a certain positive cell in $\left(G r_{k, n}\right)_{\geq 0}$.

Proposition 2.8 [13, Theorem 12.7] Let $G$ be any perfectly orientable planebipartite graph of type $(k, n)$. Then the image of the boundary measurement map $\widetilde{M} e^{2} s_{G}$ is a certain positive Grassmann cell $C_{\mathcal{M}}$ in $\left(G r_{k, n}\right)_{\geq 0}$. For every cell $C_{\mathcal{M}}$ in $\left(G r_{k, n}\right)_{\geq 0}$, there is a perfectly orientable plane-bipartite graph $G$ such that $C_{\mathcal{M}}$ is the image of $\widetilde{M} e a s_{G}$. The map $\widetilde{M} e a s_{G}$ is a fiber bundle with fiber an $r$-dimensional affine space, for some non-negative $r$. For any cell of $\left(G r_{k, n}\right)_{\geq 0}$, we can always choose a graph $G$ such that $\tilde{M}$ eas ${ }_{G}$ is a homeomorphism onto this cell.

Let us say that a plane-bipartite graph $G$ is reduced if $\tilde{M} e a s_{G}$ is a homeomorphism, and $G$ has no isolated connected components nor internal vertices incident to a single edge; see [13].

An almost perfect matching of a plane-bipartite graph $G$ is a subset $M$ of edges such that each internal vertex is incident to exactly one edge in $M$ (and the boundary vertices $b_{i}$ are incident to either one or no edges in $M$ ). There is a bijection between perfect orientations of $G$ and almost perfect matchings of $G$ where, for a perfect orientation $\mathcal{O}$ of $G$, an edge $e$ is included in the corresponding matching if $e$ is directed away from a black vertex or to a white vertex in $\mathcal{O}{ }^{4}$

For a plane-bipartite graph $G$ and the corresponding cell $C_{\mathcal{M}}=\operatorname{Image}\left(\operatorname{Meas}_{G}\right)$ in $\left(G r_{k, n}\right)_{\geq 0}$, one can combinatorially construct the matroid $\mathcal{M}$ from the graph $G$, as follows.

Proposition 2.9 [13, Proposition 11.7, Lemma 11.10] A subset $I \in\left(\begin{array}{c}{[n]} \\ k\end{array}\right)$ is a base of the matroid $\mathcal{M}$ if and only there exists a perfect orientation $\mathcal{O}$ of $G$ such that $I=I_{\mathcal{O}}$.

Equivalently, assuming that all boundary vertices $b_{i}$ in $G$ are black, $I$ is a base of $\mathcal{M}$ if and only if there exists an almost perfect matching $M$ of $G$ such that

$$
I=\left\{i \mid b_{i} \text { belongs to an edge from } M\right\} \text {. }
$$

\footnotetext{
${ }^{4}$ Note that typically $e$ is directed away from a black vertex if and only if it is directed towards a white vertex. However, we have used the word $o r$ to make the bijection well-defined when boundary vertices are not colored.
} 


\section{Toric varieties and their non-negative parts}

We may define a (generalized) projective toric variety as follows $[2,18]$. Let $S=$ $\left\{\mathbf{m}_{i} \mid i=1, \ldots, \ell\right\}$ be any finite subset of $\mathbb{Z}^{n}$, where $\mathbb{Z}^{n}$ can be thought of as the character group of the torus $\left(\mathbb{C}^{*}\right)^{n}$. Here $\mathbf{m}_{i}=\left(m_{i 1}, m_{i 2}, \ldots, m_{i n}\right)$. Then consider the map $\phi:\left(\mathbb{C}^{*}\right)^{n} \rightarrow \mathbb{P}^{\ell-1}$ such that $\mathbf{x}=\left(x_{1}, \ldots, x_{n}\right) \mapsto\left[\mathbf{x}^{\mathbf{m}_{\mathbf{1}}}, \ldots, \mathbf{x}^{\mathbf{m}_{\ell}}\right]$. Here $\mathbf{x}^{\mathbf{m}_{\mathbf{i}}}$ denotes $x_{1}^{m_{i 1}} x_{2}^{m_{i 2}} \ldots x_{n}^{m_{i n}}$. We then define the toric variety $X_{S}$ to be the Zariski closure of the image of this map. We write $\tilde{\phi}$ for the inclusion of $X_{S}$ into $\mathbb{P}^{\ell-1}$ The real part $X_{S}(\mathbb{R})$ of $X_{S}$ is defined to be the intersection of $X_{S}$ with $\mathbb{R P}^{\ell-1}$; the positive part $X_{S}^{>0}$ is defined to be the image of $\left(\mathbb{R}_{>0}\right)^{n}$ under $\phi$; and the non-negative part $X_{S}^{\geq 0}$ is defined to be the closure of $X_{S}^{>0}$ in $X_{S}(\mathbb{R})$. We note for future reference that $X_{S}$, $X_{S}(\mathbb{R})$ and $X_{S}^{\geq 0}$ are unaltered by translating the set $S$ by any integer vector.

Note that $X_{S}$ is not necessarily a toric variety in the sense of [5], as it may not be normal; however, its normalization is a toric variety in that sense. See [2] for more details.

Let $P$ be the convex hull of $S$. There is a homeomorphism from $X_{\bar{S}}^{\geq 0}$ to $P$, known as the moment map. (See [5, Section 4.2, page 81] and [18, Theorem 8.4]). In particular, $X_{S}^{\geq 0}$ is homeomorphic to a closed ball. For convenience, we will also refer to $X_{S}$ as $X_{P}$.

We now prove a simple but very important lemma.

Lemma 3.1 Suppose we have a map $\Phi:\left(\mathbb{R}_{>0}\right)^{n} \rightarrow \mathbb{P}^{N-1}$ given by

$$
\left(t_{1}, \ldots, t_{n}\right) \mapsto\left[h_{1}\left(t_{1}, \ldots, t_{n}\right), \ldots, h_{N}\left(t_{1}, \ldots, t_{n}\right)\right],
$$

where the $h_{i}$ 's are Laurent polynomials with positive coefficients. Let $S$ be the set of all exponent vectors in $\mathbb{Z}^{n}$ which occur among the (Laurent) monomials of the $h_{i}$ 's, and let $P$ be the convex hull of the points of $S$. Then the map $\Phi$ factors through the totally positive part $\left(X_{P}\right)_{>0}$, giving a map $\tau_{>0}:\left(X_{P}\right)_{>0} \rightarrow \mathbb{P}^{N-1}$. Moreover $\tau_{>0}$ extends continuously to the closure to give a well-defined map $\tau_{\geq 0}:\left(X_{P}\right)_{\geq 0} \rightarrow$ $\overline{\tau_{>0}\left(\left(X_{P}\right)_{>0}\right)}$.

Proof Let $S=\left\{\mathbf{m}_{1}, \ldots, \mathbf{m}_{\ell}\right\}$. Clearly the map $\Phi$ factors as the composite map $t=$ $\left(t_{1}, \ldots, t_{n}\right) \mapsto\left[\mathbf{t}^{\mathbf{m}_{1}}, \ldots, \mathbf{t}^{\mathbf{m}_{\ell}}\right] \mapsto\left[h_{1}\left(t_{1}, \ldots, t_{n}\right), \ldots, h_{N}\left(t_{1}, \ldots, t_{n}\right)\right]$, and the image of $\left(\mathbb{R}_{>0}\right)^{n}$ under the first map is precisely $\left(X_{P}\right)_{>0}$. The second map, which we will call $\tau_{>0}$, takes a point $\left[x_{1}, \ldots, x_{\ell}\right]$ of $\left(X_{P}\right)_{>0}$ to $\left[g_{1}\left(x_{1}, \ldots, x_{\ell}\right), \ldots, g_{N}\left(x_{1}, \ldots, x_{\ell}\right)\right]$, where the $g_{i}$ 's are homogeneous polynomials of degree 1 with positive coefficients. By construction, each $x_{i}$ occurs in at least one of the $g_{i}$ 's.

Since $\left(X_{P}\right)_{\geq 0}$ is the closure inside $X_{P}$ of $\left(X_{P}\right)_{>0}$, any point $\left[x_{1}, \ldots, x_{\ell}\right]$ of $\left(X_{P}\right)_{>0}$ has all $x_{i}$ 's non-negative; furthermore, not all of the $x_{i}$ 's are equal to 0 . And now since the $g_{i}$ 's have positive coefficients and they involve all of the $x_{i}$ 's, the image of any point $\left[x_{1}, \ldots, x_{\ell}\right]$ of $\left(X_{P}\right)_{\geq 0}$ under $\tau_{>0}$ is well-defined. Therefore $\tau_{>0}$ extends continuously to the closure to give a well-defined map $\tau_{\geq 0}:\left(X_{P}\right)_{\geq 0} \rightarrow$ $\overline{\tau_{>0}\left(\left(X_{P}\right)_{>0}\right)}$.

In Section 6 we will use this lemma to prove that $\left(G r_{k, n}\right)_{\geq 0}$ is a CW complex. 


\section{Matching polytopes for plane-bipartite graphs}

In this section we will define a family of polytopes $P(G)$ associated to plane-bipartite graphs $G$.

Definition 4.1 Given an almost perfect matching of a plane-bipartite graph $G$, we associate to it the 0 - 1 vector in $\mathbb{R}^{E(G)}$ where the coordinates associated to edges in the matching are 1 and all other coordinates are 0 . We define $P(G)$ to be the convex hull of these $0-1$ vectors.

Remark 4.2 Note that more generally, we could define $P(G)$ for any graph $G$ with a distinguished subset of "boundary" vertices. Many of our forthcoming results about $P(G)$ for plane-bipartite graphs $G$ should be extendable to this generality.

Because all of the $0-1$ vectors above have the property that $\sum_{e \ni v} x_{e}=1$ for all internal vertices $v$ of $V(G)$, the polytope $P(G)$ lies in the subspace of $\mathbb{R}^{E(G)}$ defined by $\left\{\sum_{e \ni v} x_{e}=1 \mid v \in V(G)\right\}$.

We will now see how one can arrive at these polytopes in another way. Recall that for each $G$ we have the boundary measurement map $\tilde{M} e a s_{G}: \mathbb{R}_{>0}^{E(G) / V(G)} \rightarrow G r_{k, n}$. Embedding the image into projective space via the Plücker embedding, we have an explicit formula for the coordinates given by Talaska (Corollary 2.7).

In the following definition, we use the notation of Theorem 2.6.

Definition 4.3 Fix a perfect orientation $\mathcal{O}$ of $G$. We define $P(G, \mathcal{O})$ to be the convex hull of the exponent vectors of the weights of all flows starting at $I_{\mathcal{O}}$. A priori this polytope lies in $\mathbb{R}^{E(G)}$, but we will see that $P(G, \mathcal{O})$ lies in a subspace of $\mathbb{R}^{E(G)}$.

Remark 4.4 Note that what we are doing in Definition 4.3 is taking the convex hull of all exponent vectors which occur in the $p_{J}(A)$ from Corollary 2.7, as $J$ ranges over all subsets of columns of size $\left|I_{\mathcal{O}}\right|$.

We now relate $P(G)$ and $P(G, \mathcal{O})$. We continue to use the notion of flows introduced in shortly before Theorem 2.6.

Lemma 4.5 Fix a plane-bipartite graph $G$ and a perfect orientation $\mathcal{O}_{1}$. If we choose a flow in $\mathcal{O}_{1}$ and switch the direction of all edges in this flow, we obtain another perfect orientation. Conversely, one can obtain any perfect orientation $\mathrm{O}_{2}$ of $\mathrm{G}$ from $\mathcal{O}_{1}$ by switching all directions of edges in a flow in $\mathcal{O}_{1}$.

Proof The first claim is simple: a perfect orientation is one in which each black vertex has a unique outcoming edge and each white vertex has a unique incoming edge. If we switch the orientation of all edges along one of the paths or cycles in the flow, clearly this property will be preserved.

To see the converse, let $E^{\prime}$ denote the set of edges of $G$ in which the orientations $\mathcal{O}_{1}$ and $\mathcal{O}_{2}$ disagree. It follows from the definition of perfect orientation that every edge $e$ in $E^{\prime}$ incident to some vertex $v$ can be paired uniquely with another edge $e^{\prime}$ 
in $E^{\prime}$ which is also incident to $v$ (note that at each vertex $v$ of $G$ there are either 0 or 2 incident edges which are in $E^{\prime}$ ). This pairing induces a decomposition of $E^{\prime}$ into a union of vertex-disjoint (undirected) cycles and paths. Moreover, each such cycle or path is directed in both $\mathcal{O}_{1}$ and $\mathcal{O}_{2}$ (but of course in opposite directions). This set of cycles and paths is the relevant flow.

Because of the bijection between perfect orientations and almost perfect matchings (see Section 2), Lemma 4.5 implies the following.

Corollary 4.6 Fix $G$ and a perfect orientation $\mathcal{O}$. Flows in $\mathcal{O}$ are in bijection with perfect orientations of $G$ (obtained by reversing all edges of the flow in $\mathcal{O}$ ) which are in bijection with almost perfect matchings of $G$.

We can now see the following.

Corollary 4.7 For any perfect orientation $\mathcal{O}$, the polytope $P(G, \mathcal{O})$ is a translation of $P(G)$ by an integer vector.

Proof Let $F$ denote the empty flow on $\mathcal{O}, F^{\prime}$ be some other flow in $\mathcal{O}$, and $\mathcal{O}^{\prime}$ the perfect orientation obtained from $\mathcal{O}$ by reversing the directions of all edges in $F^{\prime}$. Let $M$ and $M^{\prime}$ be the almost perfect matchings associated to $\mathcal{O}$ and $\mathcal{O}^{\prime}$. Let $x(F), x\left(F^{\prime}\right)$, $x(M)$, and $x\left(M^{\prime}\right)$ be the vectors in $\mathbb{R}^{E(G)}$ associated to this flow and these perfect orientations. Of course $x(F)$ is the all-zero vector. We claim that $x\left(M^{\prime}\right)-x(M)=$ $x(F)-x\left(F^{\prime}\right)$.

Fix an edge $e$ of $G$ : we will check that the $e$-coordinates of $x\left(M^{\prime}\right)-x(M)$ and $x(F)-x\left(F^{\prime}\right)$ are equal. First, suppose that $e$ does not occur in $F^{\prime}$. Then either $e$ appears in both $M$ and $M^{\prime}$, or in neither. So $x(F)_{e}=x\left(F^{\prime}\right)_{e}=0$ and either $x(M)_{e}=x\left(M^{\prime}\right)_{e}=0$ or $x(M)_{e}=x\left(M^{\prime}\right)_{e}=1$. Now, suppose that $e$ occurs in $F^{\prime}$, and is oriented from its white to its black endpoint in $\mathcal{O}$. So $x(F)_{e}=0$ and $x\left(F^{\prime}\right)=1$. The edge $e$ occurs in the matching $M^{\prime}$ and not in the matching $M$, so $x(M)_{e}=0$ and $x\left(M^{\prime}\right)_{e}=1$. Finally, suppose $e$ occurs in $F^{\prime}$, and is oriented from its black to its white endpoint in $\mathcal{O}$. Then $x(F)_{e}=0$ and $x\left(F^{\prime}\right)=-1$. The edge $e$ occurs in the matching $M$ and not in the matching $M^{\prime}$, so $x(M)_{e}=1$ and $x\left(M^{\prime}\right)_{e}=0$.

In particular, up to translation, $P(G, \mathcal{O})$ does not depend on $\mathcal{O}$. Recall that translating a polytope does not affect the corresponding toric variety.

In Figure 1, we fix a plane-bipartite graph $G$ corresponding to the cell of $\left(G r_{2,4}\right)_{\geq 0}$ such that the Plücker coordinates $P_{12}, P_{13}, P_{14}$ are positive and all others are 0 . We display the three perfect orientations and the vertices of $P(G)$.

In Figure 2, we fix a plane-bipartite graph $G$ corresponding to the cell of $\left(G r_{2,4}\right)_{\geq 0}$ such that the Plücker coordinates $P_{12}, P_{13}, P_{24}, P_{34}$ are positive while $P_{14}$ and $P_{23}$ are 0 . We display the four perfect orientations and the vertices of $P(G)$.

In Figure 3 we have fixed a plane-bipartite graph $G$ corresponding to the topdimensional cell of $\left(G r_{2,4}\right)_{\geq 0}$. $G$ has seven perfect orientations. We have drawn the edge graph of the four-dimensional polytope $P(G)$. This time we have depicted the vertices of $P(G)$ using matchings instead of perfect orientations. Next to each matching, we have also listed the source set of the corresponding perfect orientation. 
Fig. 1

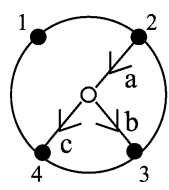

$(1,0,0)$

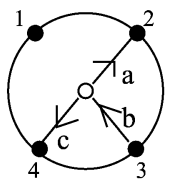

$(0,1,0)$

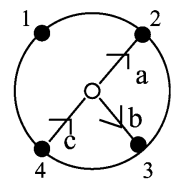

$(0,0,1)$

Fig. 2

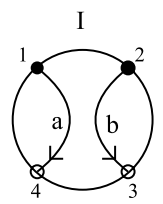

$(1,1)$

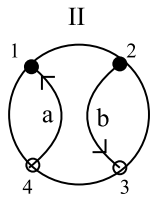

$(0,1)$

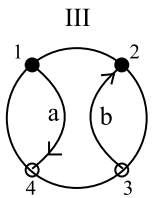

$(1,0)$
IV

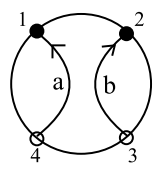

$(0,0)$

Fig. 3

12

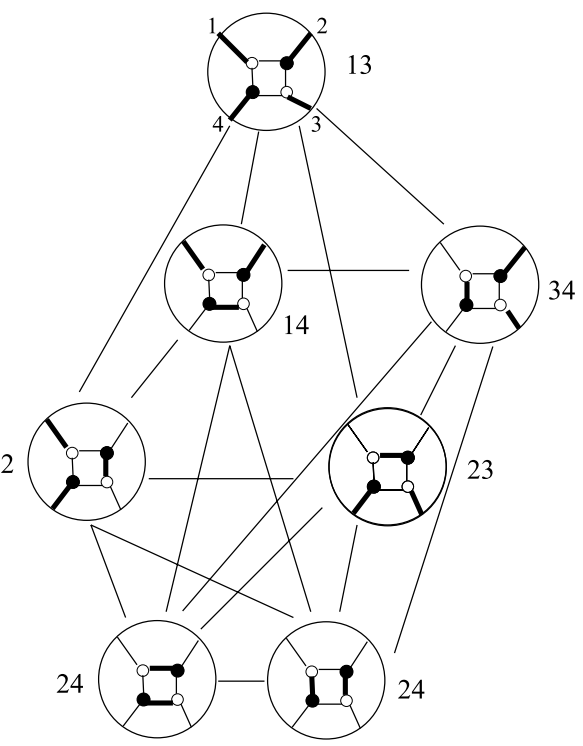

\section{Connections with matroid polytopes and cluster algebras}

Every perfectly orientable plane-bipartite graph encodes a realizable positroid, that is, an oriented matroid in which all orientations are positive. The bases of the positroid associated to a plane-bipartite graph $G$ of type $(k, n)$ are precisely the $k$-element subsets $I \subset[n]$ which occur as source sets of perfect orientations of $G$. This is easy to see, as each perfect orientation of $G$ gives rise to a parametrization of the cell $\Delta_{G}$ of $\left(G r_{k, n}\right)_{\geq 0}$ in which the Plücker coordinate corresponding to the source set $I$ is 1 . Furthermore, if one takes a (directed) path in a perfect orientation $\mathcal{O}$ and switches the orientation of each of its edges, this encodes a basis exchange.

Given this close connection of perfectly orientable plane-bipartite graphs to positroids, it is natural to ask whether there is a connection between our polytopes 
$P(G)$ and matroid polytopes. We first recall the definition of a matroid polytope. Let $M$ be a matroid of rank $k$ on the ground set $[n]$. The matroid polytope $Q(M)$ is the convex hull of the vectors $\{e(J) \mid J$ is a basis of $M\}$ where $e(J)$ is the $0-1$ vector in $\mathbb{R}^{n}$ whose $i$ th coordinate is 1 if $i \in J$ and is 0 otherwise [7]. The vertices are in one-to-one correspondence with bases of $M$. This polytope lies in the hyperplane $x_{1}+\cdots+x_{n}=0$ and, if the matroid $M$ is connected, has dimension $n-1$.

Proposition 5.1 There is a linear projection $\Psi$ from $P(G)$ to $Q\left(M_{G}\right)$. The fibers of this projection over the vertices of $Q\left(M_{G}\right)$ are the Newton polytopes for the Laurent polynomials which express the Plücker coordinates on $X_{G}$ in terms of the edge variables.

Proof If $G$ is a plane-bipartite graph of type $(k, n)$, one can associate to each vertex $v_{M}$ of $P(G)$ the basis of the corresponding positroid corresponding to the boundary edges which are matched in $G$. In terms of the bijection between perfect matchings and perfect orientations, this is the source set of the corresponding perfect orientation. This gives the linear projection $\Psi$ from $P(G)$ to $Q\left(M_{G}\right)$. To see that the statement about the fibers is true, see Corollary 2.7, and remember the relationship between matchings and flows.

The second and third authors, in [19], related the Newton polytopes of Proposition 5.1 to the positive part of the tropical Grassmannian; our results in that paper can be summarized by saying that the positive part of the tropical Grassmannian is combinatorially isomorphic to the dual fan of the fiber polytope of the map $P(G) \rightarrow Q\left(M_{G}\right){ }^{5}$

The fact that the Plücker coordinates on $X_{G}$ can all be expressed as Laurent polynomials in the edge weights is not simply a fortunate coincidence, but is a consequence ${ }^{6}$ of the fact that the coordinate ring of $X_{G}$ has the structure of a cluster algebra. (See [4] for the definition of cluster algebras, [17] for the verification that the largest cell of the Grassmannian has the structure of a cluster algebra and [13] for the fact that every $X_{G}$ has this structure.) In general, if we had a better understanding of the Newton polytopes of Laurent polynomials arising from cluster algebras, we could resolve many of the open questions in that theory.

Example 5.2 Consider the plane-bipartite graph $G$ from Figure 3. This corresponds to the positroid of rank two on the ground set [4] such that all subsets of size 2 are independent. The edge graph of the four-dimensional polytope $P(G)$ is shown in Figure 3, and each vertex is labeled with the basis it corresponds to. The matroid polytope of this matroid is the (three-dimensional) octahedron with six vertices corresponding to the two-element subsets of [4]. Under the map $\Psi$, each vertex of $P(G)$ corresponding to the two-element subset $i j$ gets mapped to the vertex of the octahedron whose $i$ th and $j$ th coordinates are 1 (all other coordinates being 0 ).

\footnotetext{
${ }^{5}$ We worked with face variables rather than edge variables in [19], but the two corresponding realizations of $P(G)$ are linearly isomorphic.

${ }^{6}$ This consequence is not completely straightforward; one must express certain ratios of the edge weights as Laurent monomials in the variables of a certain cluster, and this involves a nontrivial "chamber Ansatz".
} 


\section{$6\left(G r_{k, n}\right)_{\geq 0}$ is a CW complex}

We now prove that the cell decomposition of $\left(G r_{k, n}\right)_{\geq 0}$ is a CW complex, and obtain as a corollary that the Euler characteristic of the closure of each cell is 1 .

To review the terminology, a cell complex is a decomposition of a space $X$ into a disjoint union of cells, that is open balls. A $C W$ complex is a cell complex together with the extra data of attaching maps. More specifically, each cell in a CW complex is attached by gluing a closed $i$-dimensional ball $D^{i}$ to the $(i-1)$-skeleton $X_{i-1}$, i.e. the union of all lower dimensional cells. The gluing is specified by a continuous function $f$ from $\partial D^{i}=S^{i-1}$ to $X_{i-1}$. CW complexes are defined inductively as follows: Given $X_{0}$ a discrete space (a discrete union of 0-cells), and inductively constructed subspaces $X_{i}$ obtained from $X_{i-1}$ by attaching some collection of $i$-cells, the resulting colimit space $X$ is called a $C W$ complex provided it is given the weak topology and every closed cell is covered by a finite union of open cells.

Although we don't need this definition here, we note that a regular CW complex is a CW complex such that the closure of each cell is homeomorphic to a closed ball and the boundary of each cell is homeomorphic to a sphere. It is not known if the cell decomposition of $\left(G r_{k, n}\right)_{\geq 0}$ is regular, although the results of [22] suggest that the answer is yes.

To prove our main result, we will also use the following lemma, which can be found in $[13,15]$.

Lemma 6.1 [13, Theorem 18.3], [15, Proposition 7.2] The closure of a cell $\Delta$ in $\left(G r_{k, n}\right)_{\geq 0}$ is the union of $\Delta$ together with lower-dimensional cells.

Theorem 6.2 The cell decomposition of $\left(G r_{k, n}\right)_{\geq 0}$ is a finite $C W$ complex.

Proof All of these cell complexes contain only finitely many cells; therefore the closure-finite condition in the definition of a CW complex is automatically satisfied. What we need to do is define the attaching maps for the cells: we need to prove that for each $i$-dimensional cell there is a continuous map $f$ from $D^{i}$ to $X_{i}$ which maps $\partial D^{i}=S^{i-1}$ to $X_{i-1}$ and extends the parameterization of the cell (a map from the interior of $D^{i}$ to $X_{i}$ ).

By Corollary 2.7, if we are given a perfectly orientable plane-bipartite graph $G$, the image of the parameterization $\operatorname{Meas}_{G}$ of the cell $\Delta_{G}$ under the Plücker embedding can be described as a map $\left(t_{1}, \ldots, t_{n}\right) \mapsto\left[h_{1}\left(t_{1}, \ldots, t_{n}\right), \ldots, h_{N}\left(t_{1}, \ldots, t_{n}\right)\right]$ to projective space, where the $h_{i}$ 's are Laurent polynomials with positive coefficients. By Lemma 3.1 and Remark 4.4, the map $\operatorname{Meas}_{G}$ gives rise to a rational map $m_{G}: X_{P(G)} \rightarrow G r_{k, n}$ which is well-defined on $\left(X_{P(G)}\right)_{\geq 0}$ (a closed ball). Furthermore, it is clear that the image of $m_{G}$ on $\left(X_{P(G)}\right) \geq 0$ lies in $\left(G r_{k, n}\right)_{\geq 0}$.

Since the totally positive part of the toric variety $X_{P(G)}$ is dense in the nonnegative part, and the interior gets mapped to the cell $\Delta_{G}$, it follows that $\left(X_{P(G)}\right)_{\geq 0}$ gets mapped to the closure of $\Delta_{G}$. Furthermore, by construction, $\left(X_{P(G)}\right)_{>0}$ maps homeomorphically to the cell $\Delta_{G}$.

And now by Lemma 6.1, it follows that the boundary of $\left(X_{P(G)}\right)_{\geq 0}$ gets mapped to the $(i-1)$-skeleton of $\left(G r_{k, n}\right)_{\geq 0}$. This completes the proof that the cell decomposition of $\left(G r_{k, n}\right)_{\geq 0}$ is a CW complex. 
It has been conjectured that the cell decomposition of $\left(G r_{k, n}\right)_{\geq 0}$ is a regular $\mathrm{CW}$ complex which is homeomorphic to a ball. In particular, if a CW complex is regular then it follows that the Euler characteristic of the closure of each cell is 1 .

In [22], the third author proved that the poset of cells of $(\mathbb{G} / P)_{\geq 0}$ is thin and lexicographically shellable, hence in particular, Eulerian. In other words, the Mobius function of the poset of cells takes values $\mu(\hat{0}, x)=(-1)^{\rho(x)}$ for any $x$ in the poset. As the Euler characteristic of a finite $\mathrm{CW}$ complex is defined to be the number of even-dimensional cells minus the number of odd-dimensional cells, we obtain the following result.

Corollary 6.3 The Euler characteristic of the closure of each cell of $\left(G r_{k, n}\right)_{\geq 0}$ is 1 .

Remark After this work was compleated, Theorem 6.2 was generalized to an arbitary partial flag variety $\mathbb{G} / p$ by Rietsh and the $3^{\text {th }}$ author [16].

\section{The face lattice of $P(G)$}

We now consider the lattice of faces of $P(G)$, and give a description in terms of unions of matchings of $G$. This description is very similar to the description of the face lattice of the Birkhoff polytopes, as described by Billera and Sarangarajan [1]. In fact our proofs are very similar to those in [1]; we just need to adapt the proofs of Billera and Sarangarajan to the setting of plane-bipartite graphs.

We begin by giving an inequality description of the polytope $P(G)$.

Proposition 7.1 For any plane bipartite graph $G$, the polytope $P(G)$ is given by the following inequalities and equations: $x_{e} \geq 0$ for all edges $e$, and $\sum_{e \ni v} x_{e}=1$ for each internal vertex $v$. If every edge of $G$ is used in some almost perfect matching, then the affine linear space defined by the above equations is the affine linear space spanned by $P(G)$.

Proof Let $Q$ be the polytope defined by these inequalities. Clearly, $P(G)$ is contained in $Q$. Note that $Q$ lies in the cube $[0,1]^{E(G)}$ because if $e$ is any edge of $G$ and $v$ an endpoint of $e$ then everywhere on $Q$ we have $x_{e}=1-\sum_{e^{\prime} \ni v, e^{\prime} \neq e} x_{e} \leq 1$. Let $u$ be a vertex of $Q$. We want to show that $u$ is a $(0-1)$-vector. Suppose for the sake of contradiction that $u$ is not a $(0-1)$-vector; let $H$ be the subgraph of $G$ consisting of edges $e$ for which $0<u_{e}<1$. Note that, if $v$ is a vertex of $H$, then $v$ has degree at least 2 in $H$ since $\sum_{e \ni v} u_{e}=1$. Therefore, $H$ contains a cycle or a path from one boundary vertex of $G$ to another. We consider the case where $H$ contains a cycle, the other case is similar. Let $e_{1}, e_{2}, \ldots, e_{2 r}$ be the edges of this cycle; the length of the cycle is even because $G$ is bipartite. Define the vector $w$ by $w_{e_{i}}=(-1)^{i}$ and $w_{e}=0$ if $e \notin\left\{e_{1}, e_{2}, \ldots, e_{2 n}\right\}$. Let $\epsilon=\min _{i}\left(\min \left(u_{e_{i}}, u_{1-e_{i}}\right)\right)$. Then $u+\epsilon w$ and $u-\epsilon w$ are both in $Q$, contradicting that $u$ was assumed to be a vertex of $Q$.

Now, assume that every edge of $G$ is used in some almost perfect matching. Then $P(G)$ meets the interior of the orthant $\left(\mathbb{R}_{\geq 0}\right)^{E(G)}$, so the affine linear space spanned by $P(G)$ is the same as the affine linear space which cuts it out of this orthant. 
Corollary 7.2 Suppose that every edge of $G$ is used in some almost perfect matching. Then $P(G)$ has dimension \#Faces $(G)-1$.

Proof By proposition 7.1, the affine linear space spanned by $P(G)$ is parallel to the vector space cut out by the equations $\sum_{e \ni v} x_{e}=0$. This is precisely $H_{1}(G, \partial G)$, where $\partial G$ is the set of boundary vertices of $G$. Let $\tilde{G}$ be the graph formed from $G$ by identifying the vertices of $\partial G$. We embed $\tilde{G}$ in a sphere by contracting the boundary of the disc in which $G$ lives to a point. Then $H_{1}(G, \partial G) \cong H_{1}(\tilde{G})$, which has dimension \#Faces $(\tilde{G})-1=\# \operatorname{Faces}(G)-1$.

Note that Corollary 7.2 is correct even when some components of $G$ are not connected to the boundary, in which case some of the faces of $G$ are not discs.

\subsection{The lattice of elementary subgraphs}

Following [8], we call a subgraph $H$ of $G$ elementary if it contains every vertex of $G$ and if every edge of $H$ is used in some almost perfect matching of $H$. Equivalently, the edges of $H$ are obtained by taking a union of several almost perfect matchings of $G$. (To see the equivalence, if $\operatorname{Edges}(H)=\bigcup M_{i}$, then each edge of $H$ occurs in some $M_{i}$, which is an almost perfect matching of $H$. Conversely, if $H$ is elementary, then let $M_{1}, M_{2}, \ldots, M_{r}$ be the almost perfect matchings of $G$ contained in $H$ then, by the definition of "elementary", $\operatorname{Edges}(H)=\bigcup M_{i}$.)

The main result of this section is the following.

Theorem 7.3 The face lattice of $P(G)$ is isomorphic to the lattice of all elementary subgraphs of $G$, ordered by inclusion.

Proof We give the following maps between faces of $P(G)$ and elementary subgraphs. If $F$ is a face of $P(G)$, let $K(F)$ be the set of edges $e$ of $G$ such that $x_{e}$ is not identically zero on $F$, and let $\gamma(F)$ be the subgraph of $G$ with edge set $K(F)$. Since $F$ is a face of a $(0-1)$-polytope, $F$ is the convex hull of the characteristic vectors of some set of matchings, and $\gamma(F)$ is the union of these matchings. Thus, $F \mapsto \gamma(F)$ is a map from faces of $P(G)$ to elementary subgraphs. Conversely, if $H$ is a subgraph of $G$, let $\phi(H)=P(G) \cap \bigcap_{e \notin H}\left\{x_{e}=0\right\}$. Since $\left\{x_{e}=0\right\}$ defines a face of $P(G)$, the intersection $\phi(H)$ is a face of $P(G)$. From the description in Proposition 7.1, every face of $P(G)$ is of the form $\phi(H)$ for some subgraph $H$ of $G$. Note also that $\phi(H)=P(H)$.

We need to show that these constructions give mutually inverse bijections between the faces of $P(G)$ and the elementary subgraphs. For any face $F$ of $P(G)$, it is clear that $\phi(\gamma(F)) \supseteq F$. Suppose for the sake of contradiction that $F \neq \phi(\gamma(F))$. Then $F$ is contained in some proper face of $\phi(\gamma(F))$; let this proper face be $\phi(H)$ for some $H \subsetneq \gamma(F)$. Then there is an edge $e$ of $\gamma(F)$ which is not in $H$. By the condition that $e$ is in $\gamma(F)$, the function $x_{e}$ cannot be zero on $F$, so $F$ is not contained in $\phi(H)$ after all. We deduce that $F=\phi(\gamma(F))$.

Conversely, let $H$ be an elementary subgraph of $G$. It is clear that $\gamma(\phi(H)) \subseteq H$. Suppose for the sake of contradiction that there is an edge $e$ of $H$ which is not in 
$\gamma(\phi(H))$. Since $H$ is elementary, there is a matching $M$ of $H$ which contains the edge $e$. Let $\chi_{M}$ be the corresponding vertex of $\phi(H)$. Then $x_{e}$ is not zero on $\phi(H)$, so $e$ is in $\gamma(\phi(H))$ after all and we conclude that $H=\gamma(\phi(H))$.

The minimal nonempty elementary subgraphs of $G$ are the matchings, corresponding to vertices of $P(G)$.

Corollary 7.4 Consider a cell $\Delta_{G}$ of $\left(G r_{k, n}\right)_{\geq 0}$ parameterized by a plane-bipartite graph $G$. For any cell $\Delta_{H}$ in the closure of $\Delta_{G}$, the corresponding polytope $P(H)$ is a face of $P(G)$.

Proof By [13, Theorem 18.3], every cell in the closure of $\Delta_{G}$ can be parameterized using a plane-bipartite graph $H$ which is obtained by deleting some edges from $G$. $H$ is perfectly orientable and hence is an elementary subgraph of $G$. Therefore by Theorem 7.3, the polytope $P(H)$ is a face of $P(G)$.

\subsection{Facets and further combinatorial structure of $P(G)$}

We now give a description of the facets of $P(G)$. Let us say that two edges $e$ and $e^{\prime}$ of $G$ are equivalent if they separate the same pair of (distinct) faces $f$ and $f^{\prime}$ with the same orientation. That is, if we travel across $e$ from face $f$ to $f^{\prime}$, the black vertex of $e$ will be to our left if and only if when we travel across $e^{\prime}$ from $f$ to $f^{\prime}$, the black vertex of $e^{\prime}$ is to our left.

Lemma 7.5 If every edge of $G$ is used in an almost perfect matching then two edges $e$ and $e^{\prime}$ are equivalent if and only if the linear functionals $x_{e}$ and $x_{e^{\prime}}$ have the same restriction to $P(G)$.

Proof By Proposition 7.1, the affine linear space spanned by $P(G)$ is cut out by the equations $\sum_{e \ni v} x_{e}=1$, where $v$ runs through the internal vertices of $G$. Let $L$ be the linear space cut out by the equations $\sum_{e \ni v} x_{e}=0$; the polytope $P(G)$ is parallel to $L$ and thus the functionals $x_{e}$ and $x_{e^{\prime}}$ have the same restriction to $P(G)$ if and only if they have the same restriction to $L$. In the proof of Corollary 7.2 we identified $L$ with $H_{1}(G, \partial G)$. So we just want to determine when the restrictions of $x_{e}$ and $x_{e^{\prime}}$ to $H_{1}(G, \partial G)$ are the same.

The restrictions of $x_{e}$ and $x_{e^{\prime}}$ to $H_{1}(G, \partial G)$ are elements of the dual vector space $H^{1}(G, \partial G)$. We can identify $H^{1}(G, \partial G)$ with the vector space of functions on Faces $(G)$ summing to zero as follows: Map $\mathbb{R}^{E(G)}$ to $\mathbb{R}^{\operatorname{Faces}(G)}$ by sending an edge $e$ to the function which is 1 on one of the faces it borders and -1 on the other; the sign convention is that the sign is positive or negative according to whether $F$ lies to the right or left of $e$, when $e$ is oriented from black to white. Then $H^{1}(G, \partial G)$, which is defined as a quotient of $\mathbb{R}^{E(G)}$, is the image of this map.

We now see that $x_{e}$ and $x_{e^{\prime}}$ restrict to the same functional on $L$ if and only if they correspond to the same function on the faces of $G$. This occurs if and only if they separate the same pair of faces with the same orientation. 
Theorem 7.6 Suppose that $G$ is elementary. Then the facets of $P(G)$ correspond to the elementary subgraphs of the form $G \backslash E$, where $E$ is an equivalence class as above.

Proof First, note that if $e$ and $e^{\prime}$ are not equivalent then, by Lemma 7.5, $x_{e}$ and $x_{e^{\prime}}$ have different restrictions to $P(G)$. Thus, there is no facet of $P(G)$ on which they both vanish. On the other hand, if $e$ and $e^{\prime}$ are equivalent then, again by Lemma 7.5, on every facet of $P(G)$ where $x_{e}$ vanishes, $x_{e^{\prime}}$ also vanishes. So we see that every facet of $P(G)$ is of the form $\phi(G \backslash E)$, where $E$ is an equivalence class in $E(G)$. (Here $\phi$ is the function introduced in the proof of Theorem 7.3.)

If $\phi(G \backslash E)$ is a facet of $P(G)$ then $G \backslash E$ is elementary, by Theorem 7.3. Conversely, if $G \backslash E$ is elementary then $\phi(G \backslash E)$ is a face of $P(G)$. Since all the edges of $E$ separate the same pair of faces, $G \backslash E$ has one less face than $G$, so $\phi(G \backslash E)$ is a facet of $P(G)$, as desired.

As a special case of the preceding propositions, we get the following.

Remark 7.7 Let $N$ be a face of $P(G)$ and let $r$ be the number of regions into which the edges of $H(N)$ divide the disk in which $G$ is embedded. Then $N$ is an edge of $P(G)$ if and only if $r=2$. Equivalently, two vertices $v_{\mathcal{O}_{1}}$ and $v_{\mathcal{O}_{2}}$ of $P(G)$ form an edge if and only if $\mathcal{O}_{2}$ can be obtained from $\mathcal{O}_{1}$ by switching the orientation along a self-avoiding path or cycle in $\mathcal{O}_{1}$.

Recall that the Birkhoff polytope $B_{n}$ is the convex hull of the $n$ ! points in $\mathbb{R}^{n^{2}}$ $\left\{X(\pi): \pi \in S_{n}\right\}$ where $X(\pi)_{i j}$ is equal to 1 if $\pi(i)=j$ and is equal to 0 otherwise. It is well-known that $B_{n}$ is an $(n-1)^{2}$ dimensional polytope, whose face lattice of $B_{n}$ is isomorphic to the lattice of all elementary subgraphs of the complete bipartite graph $K_{n, n}$ ordered by inclusion [1]. Our polytopes $P(G)$ can be thought of as analogues of the Birkhoff polytope for planar graphs embedded in a disk.

Acknowledgements We are grateful to Vic Reiner for pointing out the similarity between our polytopes $P(G)$ and Birkhoff polytopes, and to Allen Knutson for many helpful conversations.

\section{Appendix A: Numerology of the polytopes $P(G)$}

In this section we give some statistics about a few of the polytopes $P(G)$. Our computations were made with the help of the software polymake [6].

Let $G 24$ denote the plane-bipartite graph from Figure 3, and let $G 25, G 26$, and $G 36$ denote the plane-bipartite graphs shown in Figures 4 . These plane-bipartite graphs give parameterizations of the top cells in $\left(G r_{2,4}\right)_{\geq 0},\left(G r_{2,5}\right)_{\geq 0},\left(G r_{2,6}\right)_{\geq 0}$, and $\left(G r_{3,6}\right)_{\geq 0}$, respectively.

The $f$-vectors of the matching polytopes $P(G 24), P(G 25), P(G 26)$ and $P(G 36)$ are $(7,17,18,8),(14,59,111,106,52,12),(25,158,440,664,590,315,98,16)$, and $(42,353,1212,2207,2368,1557,627,149,19)$ respectively. The Ehrhart series for $P(G 24), P(G 25)$ and $P(G 26)$, which give the Hilbert series of the corresponding toric varieties, are $\frac{1+2 t+t^{2}}{(1-t)^{5}}, \frac{1+7 t+12 t^{2}+4 t^{3}}{(1-t)^{7}}$, and $\frac{1+16 t+64 t^{2}+68 t^{3} 15 t^{4}}{(1-t)^{9}}$. The volumes 


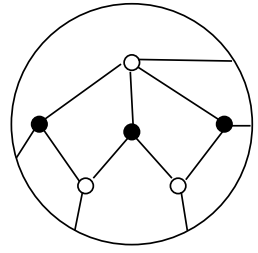

$\mathrm{G} 25$

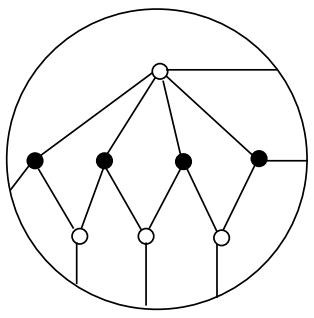

G26

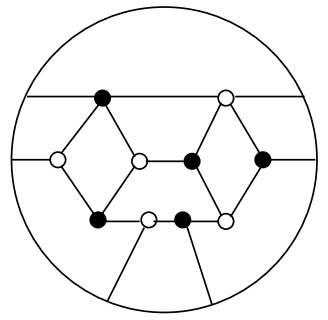

G36

Fig. 4

Fig. 5

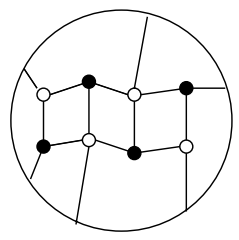

of the four polytopes are $\frac{1}{6}=\frac{4}{4 !}, \frac{1}{30}=\frac{24}{6 !}, \frac{41}{10080}=\frac{164}{8 !}$, and $\frac{781}{181440}=\frac{1562}{9 !}$. Thus, the degrees of the corresponding toric varieties are 4, 24, 164, and 1562.

Proposition A.1 Let $G 2 n$ (for $n \geq 4$ ) be the family of graphs that extend the first two graphs shown in Figure 4. Then the number of vertices of $G 2 n$ is given by $f_{0}(G 2 n)=$ $\left(\begin{array}{l}n \\ 3\end{array}\right)+n-1$.

Proof This can be proved by induction on $n$ by removing the leftmost black vertex. We leave this as an exercise for the reader.

Note that in general there is more than one plane-bipartite graph giving a parameterization of a given cell. But even if two plane-bipartite graphs $G$ and $G^{\prime}$ correspond to the same cell, in general we have $P(G) \neq P\left(G^{\prime}\right)$. For example, the plane-bipartite graph in Figure 5 gives a parameterization of the top cell of $\left(G r_{2,6}\right)_{\geq 0}$. Let us refer to this graph as $\hat{G} 26$. However, $P(\hat{G} 26) \neq P(G 26)$ : the $f$-vector of $P(\hat{G} 26)$ is $(26,165,460,694,615,326,100,16)$.

\section{References}

1. Billera, L., Sarangarajan, A.: The Combinatorics of Permutation Polytopes. DIMACS Series in Discrete Mathematics and Theoretical Computer Science, vol. 24 (1996)

2. Cox, D.: What is a toric variety? In: Topics in Algebraic Geometry and Geometric Modeling. Contemp. math., vol. 334, pp. 203-223. Amer. Math. Soc., Providence (2003)

3. Fomin, S., Shapiro, M.: Stratified spaces formed by totally positive varieties. Mich. Math. J. 48, 253270 (2000)

4. Fomin, S., Zelevinsky, A.: Cluster algebras I: foundations. J. Am. Math. Soc. 22, 497-527 (2002)

5. Fulton, W.: Introduction to Toric Varieties. Annals of Mathematics Studies, vol. 131. Princeton University Press, Princeton (1993) 
6. Gawrilow, E., Joswig, M.: Polymake: a framework for analyzing convex polytopes and simplicial complexes. http://www.math.tu-berlin.de/polymake (1997-2003). Version 2.0, with contributions by Thilo Schroder and Nikolaus Witte

7. Gelfand, I., Goresky, R., MacPherson, R., Serganova, V.: Combinatorial geometries, convex polyhedra, and Schubert cells. Adv. Math. 63(3), 301-316 (1987)

8. Lovasz, L., Plummer, M.: Matching Theory. Elsevier Science, New York (1986)

9. Lusztig, G.: Introduction to total positivity. In: Hilgert, J., Lawson, J.D., Neeb, K.H., Vinberg, E.B. (eds.) Positivity in Lie Theory: Open Problems, pp. 133-145. de Gruyter, Berlin (1998)

10. Lusztig, G.: Total positivity in partial flag manifolds. Represent. Theory 2, 70-78 (1998)

11. Lusztig, G.: Total positivity in reductive groups. In: Lie Theory and Geometry: in Honor of Bertram Kostant. Progress in Mathematics, vol. 123. Birkhauser, Basel (1994)

12. Marsh, R., Rietsch, K.: Parametrizations of flag varieties. Represent. Theory, 8 (2004)

13. Postnikov, A.: Total positivity, Grassmannians, and networks. http://front.math.ucdavis.edu/math.CO/ 0609764

14. Rietsch, K.: Total positivity and real flag varieties. PhD dissertation, MIT (1998)

15. Rietsch, K.: Closure relations for totally non-negative cells in $G / P$. Math. Res. Lett. 13(5-6), 775786 (2006)

16. Rietsh, K., Williams, L.: The totaly nonnegative part of $G / p$ is a CW complex. Transformation Groups, to appear

17. Scott, J.: Grassmannians and cluster algebras. Proc. Lond. Math. Soc. 92(2), 345-380 (2006)

18. Sottile, F.: Toric ideals, real toric varieties, and the moment map. In: Topics in Algebraic Geometry and Geometric Modeling. Contemp. Math., vol. 334, pp. 225-240 (2003)

19. Speyer, D., Williams, L.: The tropical totally positive Grassmannian. J. Algebr. Comb. 22(2), 189-210 (2005)

20. Talaska, K.: A formula for Plücker coordinates of a perfectly oriented network. arXiv:0801.4822; Int. Math. Res. Not., to appear

21. Williams, L.: Enumeration of totally positive Grassmann cells. Adv. Math. 190(2), 319-342 (2005)

22. Williams, L.: Shelling totally non-negative flag varieties. J. Reine Angew. Math. 609, 1-22 (2007) 